\title{
Galectin-3 and Severity of The Coronary Artery Disease in Ischemic Patients Guided by Coronary Angiography \\ Reda Biomy Bastawesy ${ }^{1}$, Mohamed Kamal Salama ${ }^{2}$, Hossam Eldin Mohamed Mansour'2, Ahmed Barakat Ahmed²,
}

Faculty of Medicine, Department of cardiology ${ }^{1}$ Kafr El Sheikh University, ${ }^{2}$ Aswan University

Corresponding author: Ahmed Barakat Ahmed, MSC, E-mail: ahmed8930ahmed@ gmail.com.

Telephone: 01100138516

\section{ABSTRACT}

Background: The inflammatory process is actively involved in atherosclerosis and underlies all stages of atherosclerotic plaque development: the beginning, the progression and the plaque rupture. It has been reported that during the inflammatory process the expression of Gal-3 is increased in human atherosclerotic lesions, suggesting its involvement in atherogenesis. Aim: In the present study, we tried to evaluate the levels of Gal-3 in patients with chronic stable angina and its relation to the severity of coronary artery disease (CAD) and the CAD risk factors such as Aging, Diabetes, Hypertension, Smoking and Dyslipidemia. Patients and Methods: Our study population consisted of 95 chronic stable angina patients who were planned for coronary angiography. All patients had clinical and/or electrocardiographic evidence of significant stable ischemic heart disease.

Results: We found a significant positive correlation between CAD and Gal-3 levels $(r=0.207)$, ( $p$ value $=0.045)$. Patients with multi-vessel (MVD) had significantly higher plasma Gal-3 levels and syntax score than 3 vessel disease (TVD) and single vessel disease (SVD) (P value <0.001)., while Patients with TVD had significantly higher plasma Gal-3 levels and syntax score than SVD, but still lower than MVD Patients (P value $<0.001$.

Conclusion: Gal-3 plasma levels were significantly correlated with the severity of CAD in chronic stable angina and can be used as a prognostic marker of chronic stable angina patients.

Recommendation: We recommend that the Gal-3 might be useful for risk stratification and outcome prediction of coronary heart diseases.

Keywords: Coronary artery disease, syntax score, chronic stable angina.

\section{INTRODUCTION}

The inflammatory process is actively involved in atherosclerosis and all stages of atherosclerotic plaque development: the beginning, the progression and the plaque rupture ${ }^{1}$. In recent years, several studies have been done define the mechanism that leads to acute clinical events, and systemic approaches are pursued to discover serum biomarkers useful to identify patients with plaque at risk of future vascular events ${ }^{2,3}$. Galectin-3 (Gal-3) is a galactosidase-binding protein and a member of the Galectin family which contains more than 10 members. It is expressed in the epithelia of several organs and inflammatory cells such as macrophages, dendritic cells and Kupffer cells ${ }^{4}$. The extracellular Gal-3 has been demonstrated to activate different types of inflammatory cells such as monocytes/macrophages, mast cells, neutrophils, and lymphocytes, and it has been shown to facilitate cellcell and cell-matrix interaction ${ }^{5}$. It is known that Gal3 is able to form dimers through the amino-terminal non-lectin domain that allows cross-linking appropriate glycoproteins on the cell surface ${ }^{6}$.During the inflammatory process this lectin is up regulated and it has been reported that the expression of Gal-3 is increased in human atherosclerotic plaques, suggesting its involvement in atherogenesis ${ }^{7}$. In the present study, we will try to evaluate the levels of Gal3 in patients with chronic stable angina and its relation to the severity of CAD and the coronary artery disease risk factors such as Aging, Diabetes, Hypertension, Smoking and Dyslipidemia.

\section{AIM OF THE STUDY}

we tried to evaluate the levels of Gal-3 in patients with chronic stable angina and its relation to the severity of CAD and the coronary artery disease risk factors such as Aging, Diabetes, Hypertension, Smoking and Dyslipidemia.

\section{PATIENTS AND METHODS}

Our study was conducted from January 2018 to July 2018 at Aswan university hospital. Our study population included 95 chronic stable angina patients who were planned for coronary angiography. All patients had clinical and/or electrocardiographic evidence of significant stable ischemic heart disease. Patients with Prior heart failure, Severe valvular heart diseases, Connective tissue diseases, Coexisting cancers and chemotherapy and radiotherapy, Pericardial disease, Cirrhosis and Atrial fibrillation were excluded from the study.

All the patients were subjected to

A) Clinical assessment including ( $\mathrm{CV}$ risk factors, general and local examination).

B) Electrocardiography twelve leads resting ECG done for all patients to exclude non-sinus rhythm and to detect any ischemic changes or arrhythmias.

C) Echocardiography done in the left lateral position using Philips IE 33 X51 MHz transducer on outpatient basis, m-mode and $2 \mathrm{D}$ was acquired to evaluate 
systolic and diastolic function, regional wall motion abnormalities and left ventricular ejection fraction, and also to exclude other causes of chest pain ${ }^{8}$.

D) Peripheral Blood samples to measure the following parameters (random blood glucose, renal function, lipid profile and Plasma Gal-3). Peripheral blood samples and plasma Gal-3 assay were gathered within 24 hours from admission. Peripheral blood samples were collected in test tubes containing Ethylene diamine tetra acetic Acid (EDTA) and heparin. The EDTA blood sample was centrifuged $1000 \mathrm{xg}$ for 15 minutes and plasma was frozen at $-20 \mathrm{c}$. Plasma concentrations of Gal-3 were detected by an enzymelinked immunosorbent assay kit (Bioscience, American) $)^{9}$.

E) Coronary angiography for diagnosis of CAD Coronary angiography done using a modified Seldinger's technique; an 18-gage thin-walled needle is inserted at a 30- to 45- degree angle into the femoral artery, and a 0.035 or 0.038 in. J-tip Teflon-coated guide wire is advanced through the needle into the artery ${ }^{10}$. an arterial sheath with proximal one-way valve and side arm is placed over the wire and allows multiple catheter exchanges. Sheath diameter is at least equal to coronary catheter diameter (typically 6 French sheaths are used). The Judkins left 4 (JL4) and JL3.5 were used to engage the left coronary system and The Judkins right 4 (JR4) catheter is used in the majority of cases to access the right coronary artery. After finishing the procedure, manual compression is most commonly used for hemostasis after removal of the indwelling femoral artery sheath ${ }^{10}$.Assessment of the severity of the CAD was done by using the syntax score.
SYNTAX score was calculated retrospectively based on diagnostic angiograms obtained before the PCI by two experienced interventional cardiologists using the SYNTAX score calculator (available at http://www.syntaxscore.com). In case of disagreement, the opinion of a third observer was obtained, and the final decision was made by consensus ${ }^{11}$.

\section{Statistical analysis}

The data were tested for normality using the Anderson-Darling test and for homogeneity variances prior to further statistical analysis. Categorical variables were described by number and percent $(\mathrm{N}$, $\%$ ), where continuous variables described by mean and standard deviation (Mean, SD). Chi-square test and fisher exact test used to compare between categorical variables where compare between continuous variables by t-test and IndependentSamples T test ANOVA. A two-tailed $\mathrm{p}<0.05$ was considered statistically significant. We are used person and spearman correlation to appear the association between variables. All analyses were performed with the IBM SPSS 20.0 software $^{12}$.

\section{RESULTS}

Our study included 95 patients that were diagnosed as chronic stable angina and were planned for coronary angiography between $1^{\text {st }}$ January 2018 and $31^{\text {th }}$ July 2018 . Their mean age was $(57.05 \pm 7.77)$ ranges from 37 to 68 years, $62.1 \%$ (59) were males and $37.9 \%$ (36) were females (table 1).

Demographic characteristics of the studied population: (Table 1)

\begin{tabular}{|c|c|c|}
\hline \multicolumn{2}{|l|}{ Variables } & Patients(n=95) \\
\hline Age (years) & Mean \pm SD & $57.05 \pm 7.77$ \\
\hline Sex & $\begin{array}{l}M(n, \%) \\
F(n, \%)\end{array}$ & \begin{tabular}{|l|l}
$59(62.1 \%)$ \\
$36(37.9 \%)$ \\
\end{tabular} \\
\hline Hypertension & $\begin{array}{l}\text { Yes }(n, \%) \\
\text { No }(n, \%)\end{array}$ & $\begin{array}{r}59(62.1 \%) \\
36(37.9 \%) \\
\end{array}$ \\
\hline Diabetes & $\begin{array}{l}\text { Yes }(n, \%) \\
\text { No }(n, \%) \\
\end{array}$ & $\begin{array}{l}51(53.7 \%) \\
44(46.3 \%) \\
\end{array}$ \\
\hline Smoking & $\begin{array}{l}\text { Yes }(n, \%) \\
\text { No }(n, \%) \\
\end{array}$ & $\begin{array}{l}59(62.1 \%) \\
36(37.9 \%) \\
\end{array}$ \\
\hline Positive family history for CAD & $\begin{array}{l}\text { Yes }(n, \%) \\
\text { No }(n, \%) \\
\end{array}$ & $\begin{array}{l}33(34.7 \%) \\
62(65.3 \%) \\
\end{array}$ \\
\hline Dyslipidemia & $\begin{array}{l}\text { Yes }(n, \%) \\
\text { No }(n, \%)\end{array}$ & $\begin{array}{l}34(35.8 \%) \\
61(64.2 \%)\end{array}$ \\
\hline Total cholesterol, mg/dL & Mean \pm SD & $206.33 \pm 36.75$ \\
\hline HDL-C, mg/dL & Mean \pm SD & $41.85 \pm 10.08$ \\
\hline LDL-C, mg/dL & Mean \pm SD & $128.49 \pm 33.3$ \\
\hline
\end{tabular}

HDL-C=High density lipoprotein cholesterol, LDL-C= low density lipoprotein cholesterol, SD= standard deviation. 
Every patient in our study was subjected to clinical, laboratory and echocardiographic evaluation. Table 2 shows the measurement of interest in our study including BMI $(\mathrm{kg} / \mathrm{m} 2)$, creatinine clearance, galectin-3 (ng/mL), LVEF (\%). Mean LVEF was (56 \pm 70$)$ and the level of Gal-3 ranged from 1.77 to $23.4 \mathrm{ng} / \mathrm{mL}$, with a median of $7.88 \mathrm{ng} / \mathrm{mL}$ (Table 2).

Clinical, Laboratory and echocardiographic data of the studied patients: (Table 2)

\begin{tabular}{|c|l|c|}
\hline \multicolumn{2}{|c|}{ Variables } & Patients(n=95) \\
\hline Galectin-3 $(\mathrm{ng} / \mathrm{mL})$ & Mean \pm SD & $\begin{array}{c}10.1 \pm 7.08 \\
\mathbf{7 . 8 8}(\text { Median })\end{array}$ \\
\hline \hline Syntax score & Mean \pm SD & $15.97 \pm 7.87$ \\
\hline LVEF $(\%)$ & Mean \pm SD & $\mathbf{5 6 \%} \pm 70 \%$ \\
\hline Cr. Cl & Mean \pm SD & $\mathbf{8 6 . 3 2} \pm 7.18$ \\
\hline BMI $(\mathrm{kg} / \mathrm{m} 2)$ & Mean \pm SD & $\mathbf{2 6 . 9 5} \pm 2$ \\
\hline
\end{tabular}

$\mathrm{SD}=$ standard deviation, $\mathrm{Cr} . \mathrm{Cl}=$ creatinine clearance, $\mathrm{LVEF}=$ left ventricular ejection fraction, $\mathrm{BMI}=$ body mass index.

In the CAD population, Plasma concentrations of Gal-3 levels were positively Correlated with hypertension (Fig1), while showed non-significant correlation with diabetes Mellitus, Cr. Cl, BMI, smoking, age, and LDL-C (Table3).

Correlation between Gal-3 CV risk factors: (table3).

\begin{tabular}{|c|c|c|}
\hline Variables & r & P value \\
\hline Hypertension & $\mathbf{0 . 3 1 8}$ & $\mathbf{0 . 0 0 2}$ \\
\hline Diabetes & $-\mathbf{0 . 0 5 1}$ & $\mathbf{0 . 6 2 3}$ \\
\hline Smoking & $\mathbf{0 . 0 5 8}$ & $\mathbf{0 . 5 7 4}$ \\
\hline Age & $\mathbf{0 . 0 2 6}$ & $\mathbf{0 . 8 0 1}$ \\
\hline BMI & $\mathbf{- 0 . 0 5 6}$ & $\mathbf{0 . 5 8 9}$ \\
\hline LDL-C & $\mathbf{0 . 0 8 7}$ & $\mathbf{0 . 4 0 3}$ \\
\hline Cr. Cl & $\mathbf{- 0 . 0 1 4}$ & $\mathbf{0 . 8 9 3}$ \\
\hline
\end{tabular}

* Statistically significant correlation $(\mathbf{p}<0.05), * *$ statistically significant correlation $(\mathbf{p}<0.01) \mathbf{C r}$. $\mathbf{C l}=$ creatinine clearance, BMI = body mass index, LDL-C = low density lipoprotein cholesterol.

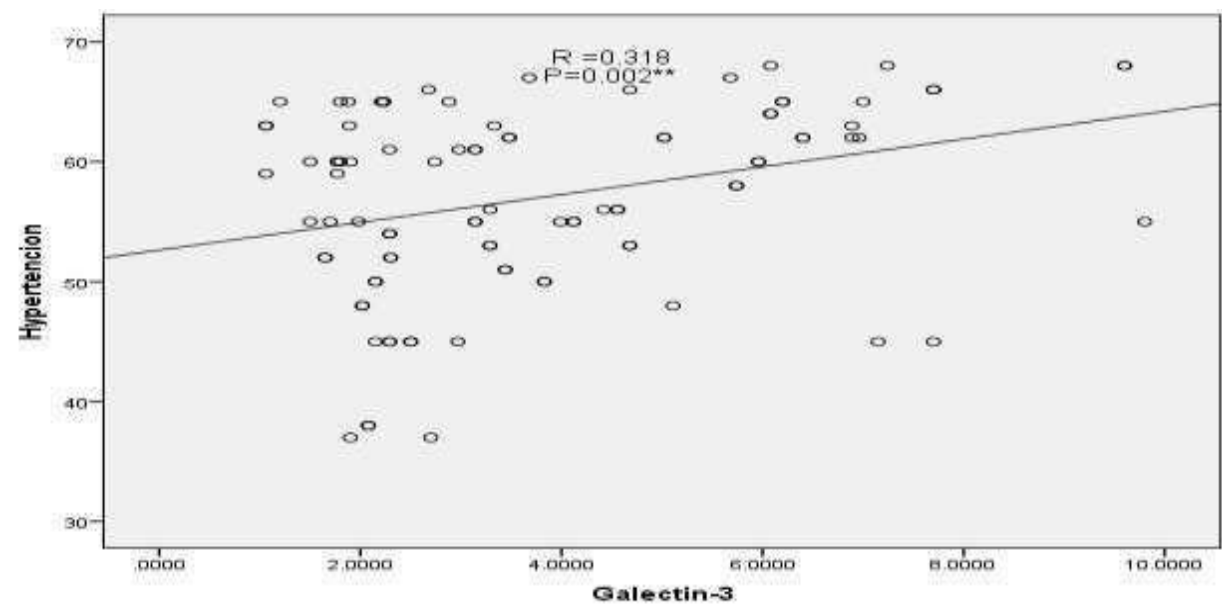

(Fig1) Correlation between Gal-3 and hypertension

Every patient in our study was subjected to coronary angiographic evaluation. Single vessel disease was present in 31 patients (32.6\%), two vessel diseases was present in 24 patients $(25.3 \%)$ and three vessel diseases was present in 40 patients $(42.1 \%)$. There was a significant positive correlation between severity of CAD 
Galectin-3 and Severity of The Coronary Artery Disease...

(assessed by syntax score) and Gal-3 ( $\mathrm{r}=0.915)$, ( $\mathrm{p}$ value $<0.001)$ (Fig2). Patients with MVD had significantly higher plasma Gal-3 levels and syntax score than TVD and SVD, (P value <0.001). Patients with TVD had significantly higher plasma Gal-3 levels and syntax score than SVD, but still lower than MVD, (P value <0.001). (Table4).

Comparison between numbers of coronary vessels affected, Gal-3 and syntax score (Table4)

\begin{tabular}{|c|c|c|c|c|}
\hline & \multicolumn{3}{|c|}{ CAD } & \multirow[t]{3}{*}{ P. value } \\
\hline & MVD & SVD & TVD & \\
\hline & Mean \pm SD & Mean \pm SD & Mean \pm SD & \\
\hline SYNTAX score & $23.68 \pm 5.27$ & $8.39 \pm 2.89$ & $12.92 \pm 2.15$ & $<0.001 * *$ \\
\hline Gal-3 & $17.5 \pm 3.93$ & $2.96 \pm 0.95$ & $6.99 \pm 1.78$ & $<0.001 * *$ \\
\hline Median & 17.690 & 2.980 & 6.925 & \\
\hline
\end{tabular}

One-way ANOVA test, $*$ statistically significant difference $(\mathrm{p}<0.05), * *$ highly significant difference $(\mathrm{p}<0.01)$. $\mathrm{SVD}=$ single vessel disease, $\mathrm{TVD}=$ two vessel disease, $\mathrm{MVD}=$ multi vessel disease, $\mathrm{SD}=$ standard deviation, $\mathrm{CAD}=$ coronary artery disease.

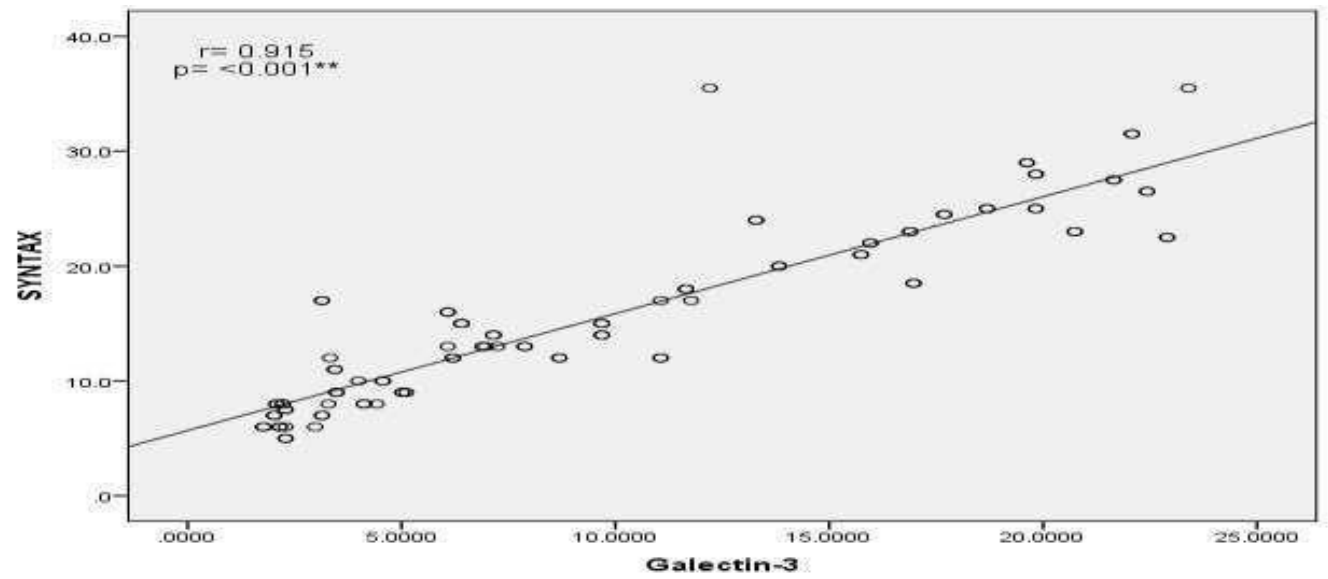

between Gal- 3 and syntax score.

(Fig2) Correlation

In our study LVEF was measured by transthoracic echocardiography using m-mode method and correlated with Gal-3 and we found that there was no significant correlation between Gal-3 and left ventricular ejection fraction $(\mathrm{r}=-0.14)$, $(\mathrm{p}$ value $=0.895)$. $(\mathrm{Fig} 3)$.

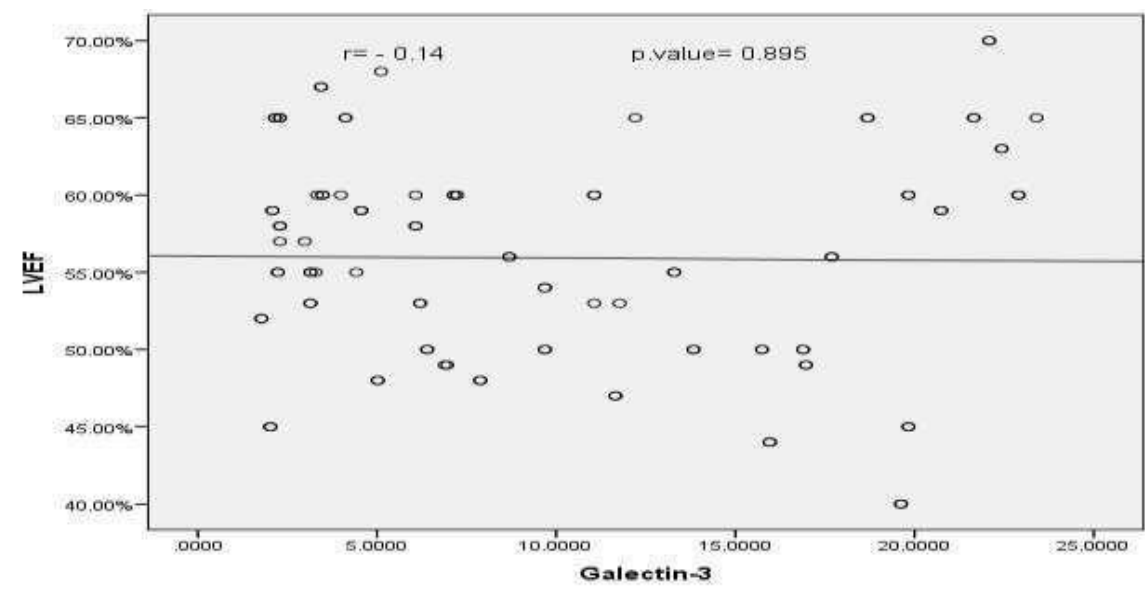

(Fig3) Correlation between Gal-3 and LVEF 


\section{DISCUSSION}

Several independent pathways of evidence suggest inflammation as a key regulatory process that links the atherosclerotic risk factors and its complications with altered arterial biology. This new evidence regarding the pathophysiology of atherosclerosis has provided clinical insight and practical tools that may concur in patient management ${ }^{13}$. In the present observational prospective study, we tried to evaluate the levels of Gal-3 in patients with chronic stable angina and its relation with the severity of CAD and the coronary artery disease risk factors such as Aging, Diabetes, Hypertension, Smoking and Dyslipidemia.

\section{Correlation between Gal-3 and CV risk factors:}

Our study showed that plasma concentrations of Gal-3 levels was positively correlated with hypertension, while showed nonsignificant correlation with diabetes Mellitus, $\mathrm{Cr}$. $\mathrm{Cl}$, BMI, age, smoking, and LDL-C in the CAD population ${ }^{14}$. Our findings are in agreement with Yao who showed that serum Gal-3levels increase in patients with hypertension; however, it is more obvious in patients with left ventricular hypertrophy. Therefore, Gal-3 is independently correlated with left Ventricular remodeling (LVR) and can be regarded as a valuable biomarker for early Cardiac remodeling ${ }^{15}$. These findings may be explained by the fact that Hypertension is normally a chronic medical condition characterized by elevated arterial blood pressure. Hypertensive cardiac remodeling begins with inflammation, an increased deposition of extracellular matrix proteins, followed by formation of myocardial fibrosis and finally cardiac dysfunction ${ }^{16}$.

\section{Correlation between Gal-3 and CAD:}

There is a significant positive correlation between CAD and Gal-3 ( $\mathrm{r}=0.207)$, ( $\mathrm{p}$ value= 0.045). Our findings are also in agreement with Kusaka who observed that the higher concentrations of galectin-3 in CAD patients ${ }^{17}$. Our findings are also in agreement with Aksan who found that the serum Gal-3 level had a positively correlated with the severity of coronary artery stenosis, number of vessels involved and serum $\mathrm{C}$-reactive protein levels ${ }^{18}$. These findings may be explained by the fact that Gal-3, a major inflammatory signal and promoter, can activate reduced-coenzyme, increase neutrophil superoxide production, stimulate outbreak of the respiratory chain and trigger oxidative-stress reactions leading to an increase in uptake of ox-LDL by vascular endothelial cells, macrophages and smooth muscle cells, eventually causing the proliferation of foam cells and promoting atherosclerosis ${ }^{19}$.

\section{Comparison between numbers of coronary vessels affected, Syntax score and Gal-3:}

Patients with MVD had significantly higher plasma Gal-3 levels and syntax score than TVD and SVD, (P value <0.001). Patients with TVD had significantly higher plasma Gal-3 levels and syntax score than SVD, but still lower than MVD, (P value $<0.001)^{20}$.

Our findings are in agreement with Sanchez-Mas who found that Patients with three vessel disease had higher levels of Gal-3 than patients with 1- or 2vessel disease. Gal-3 levels were significantly higher in patients diagnosed with $\mathrm{MI}$ or CHD compared to control ${ }^{21,22}$. Our findings are in agreement with Falcone who found that patients with three vessel disease had higher levels of Gal-3 than patients with 1 - or 2-vessel disease ${ }^{23}$. Our findings are also in agreement with the study of $\mathbf{T}$ sail who observed that multivessel coronary patients had higher level of Gal-3 than control group, and multi vessel disease correlates with higher Gal-3 level and WBC count. There is a significant association between Gal-3 and number of affected coronary blood vessels ${ }^{24}$.

\section{Correlation between Gal-3 and left ventricular ejection fraction:}

There is no significant correlation between Gal-3 and left ventricular ejection fraction $(\mathrm{r}=$ $0.14)$, $(\mathrm{p}$ value $=0.895)$. These findings may be explained by the fact that Gal-3 has been proposed as a biomarker involved in the pathophysiology of $\mathrm{HF}$ and may be of lesser importance during the early stage of disease ${ }^{25,26}$. In HF, Gal-3 concentration is associated with echocardiographic marker of ventricular ejection fraction ${ }^{28}$. The left ventricular ejection fraction in our patients was comprised in normal range and we did not observe a relation between Gal-3 and left ventricular ejection fraction. Recently, other studies have reported the absence of this relation and in particular, no association between $\mathrm{LVEF} \geq 40 \%$ and value of plasma Gal-3 levels was observed ${ }^{27,28}$.

\section{CONCLUSION}

Gal-3 plasma levels are significantly correlated with the severity of CAD in chronic stable angina and can be used as a prognostic marker of chronic stable angina patients. 


\section{RECOMMENDATION}

We recommend that the Gal-3 might be useful for risk stratification and outcome prediction of coronary heart diseases.

\section{REFERENCES}

1. Libby P, Ridker PM, Hansson GK (2009): Inflammation In atherosclerosis: from pathophysiology to practice. JAm Coli Cardiol.,54:2129-38.

2. Uno K, Nicholls SJ (2010): Biomarkers of inflammation and oxidative stress in atherosclerosis. Biomark Med., 4:361-73.

3. Drakopoulou M, Toutouzas K, Stefanadi E, Tsiamis E, Tousoulis D, Stefanadis C (2009): Association of inflammatory markers with angiographic severity and extent of coronary artery disease. Atherosclerosis, 206:335-9.

4. Sano H, Hsu DK, Apgar JR, Yu L, Sharma BB, Kuwabara I, Izui S, Liu FT (2003): Critical role of galectin-3 in phagocytosis by macrophages. J Clin Invest.,112:389-97.

5. Liu FT, Patterson RJ, Wang JL (2002): Intracellular functions of galectins. Biochim Biophys Acta, 1572:263-73.

6. Ochieng J, Furtak V, Lukyanov $P$ (2004): Extracellular functions of galectin-3. Glycoconj J., 19:52735.

7. Nachtigal M, AI-Assaad Z, Mayer EP, Kim K, Monsigny M (1998): Galectin-3 expression in human atherosclerotic lesions. Am J Pathol.,152:1199208.

8. Douglas PS, Garcia MJ, Haines DE, Lai WW, Manning WJ, Patel AR, Picard MH, Polk DM, Ragosta M, Ward RP, Weiner RB (2011): ACCF/ASE/AHA/ASNC/HFSA/HRS/SCAI/SCCM/ SCCT/SCMR 2011 Appropriate Use Criteria for Echocardiography. Physicians. J Am Coll Cardiol.,57:1126-1166.

9. Christenson RH, Duh SH, Wu AH, et al (2010): Multi-center determination of galectin-3 assay performance characteristics: anatomy of a novel assay for use in heart failure Clin Biochem., 43:683-690.

10.Powell D , Moxey CF (2011): Diagnostic Catheterization, In: Invasive Cardiology: a manual for cath lab personnel, 3rd Edition, Watson S \& Gorski KA, pp. 143-162, Jones \& Bartlett Learning, , USA.

11. Genereux P, Palmerini T, Caixeta A (2011): Syntax score reproducibility and variability between interventional cardiologists, core laboratory technicians, and quantitative coronary measurements, Circ Cardiovasc Interv.,4:553-61.

12.http://www.ibm.com/support

13.Libby P, Ridker PM, Hansson GK (2009): Leducq Transatlantic Network on Atherothrombosis. Inflammation In atherosclerosis: from pathophysiology to practice. JAm Coli Cardiol.,54:2129-38.
14.U.S. Preventive Services Task Force (2003): Screening for obesity in adults: Recommendations and rationale. Ann Intern Med., 139:930-2.

15. Cha J H, Wee $\mathbf{H} \mathbf{J}$, Seo J H (2014): AKAP12 mediates barrier functions of fibrotic scars during CNS repair. PLoS One, 9(4): e94695.

16. Yao Y, Shen D, Chen R (2015): Galectin-3 Predicts Left Ventricular Remodeling of Hypertension. Int J Cardiol., 176(3):1423-1425.

17. Aksan G, Gedikli O, Keskin K (2016): Is galectin-3 a biomarker, aplayeror both-in the presence of coronary atherosclerosis? J Investig Med., 64(3):764770 .

18. Kusaka H, Yamamoto E, Hirata Y (2015): Clinical significance of plasma Gal-3 in patients with coronary artery disease. Int J Cardiol., 201: 532-534.

19. Fernandes B A, Campanhole G, Wang P H (2008): A Role for galectin-3 in renal tissue damage triggered by ischemia and reperfusion injury. Transpl Int., 21(10):999-1007.

20.Lama More RA, Alonso Franch A, Gil-Campos M (2006): Childhood obesity. Recommendations of the nutrition committee of the Spanish association of pediatrics. Part I. Prevention Early Detection Role of the pediatrician. A Pediatr (Barc), 65:607- 15.71.

21. Sanchez-Mas J, Lax A, Asensio-Lopez M C (2014): Galectin-3 expression in cardiac remodeling after myocardial infarction. Int J Cardiol., 172(1): e98e101.

22.Lisowska A, Knapp M, Tycinska A (2016): Predictive value of Galectin-3 for the occurrence of coronary artery disease and prognosis after myocardial infarction and its association with carotid IMT values in these patients: A mid-term prospective cohort study. Atherosclerosis, 246:309-317.

23. Falcone C, Lucibello S, Mazzucchelli I (2011): Galectin-3 plasma levels and coronary artery disease: a new possible biomarker of acute coronary syndrome, Int J Immunopathol Pharmacol., 24 (4): 905-913.

24. Tsai, T, Sung P, Chang L (2012): Value and Level of Galectin-3 in Acute Myocardial Infarction Patients Undergoing Primary Percutaneous Coronary Intervention. Journal of Atherosclerosis and Thrombosis, 19: 1073-1082.

25.De Boer RA, Yu L, van Veldhuisen DJ (2010): Galectin-3 in cardiac remodeling and heart failure. Cur Heart Fail Rep., 7:1-8.

26. Christenson RH, Duh SH, Wu AH (2010): Multicenter determination of galectin-3 assay performance characteristics: Anatomy of a novel assay for use in heart failure Clin Biochem., 43:683-90.

27.De Boer RA, Lok DJ, Jaarsma T (2011): Predictive value of plasma galectin-3 levels in heart failure with reduced and preserved ejection fraction. Ann Med., 43:60-68.

28. Tang WH, Shrestha K, Shao Z (2009): Usefulness of plasma galectin-3 levels in systolic heart failure to predict renal insufficiency and survival. Am J Cardiol $., 108: 385-90$. 